\title{
Mitral Valve Surgery via a Right Infra-axillary Thoracotomy in High-Risk Reoperative Patients
}

\author{
Hailong Cao, MD, PhD, Qing Zhou, MD, Yunxing Xue, MD, Fudong Fan, MD, Dongjin Wang, MD, PhD \\ Department of Thoracic and Cardiovascular Surgery, the Affiliated Drum Tower Hospital of Nanjing University Medical School, \\ Nanjing, China
}

\section{ABSTRACT}

Background: A right infra-axillary thoracotomy can offer excellent exposure of the mitral valve. This study evaluated this incision for high-risk patients undergoing redo mitral valve procedures.

Methods: Of a series of 189 patients who had redo mitral valve surgery, 32 were reoperated via vertical infra-axillary thoracotomy based on previous aortic valve replacement, dense adhesion, location of patent bypass grafts, and peripheral vascular disease.

Results: Sternotomy was avoided in all cases. The mitral valve was replaced in 22 patients and repaired in 10 patients; left atrial folding was performed in 6 patients. All patients had uneventful outcomes and normal valve function during follow-up.

Conclusions: Reoperative mitral valve surgeries can be performed safely using right infra-axillary thoracotomy in certain patients. The procedure offers excellent exposure of the mitral valve and minimizes the need for cardiac dissection, thus reducing injury risk. Avoiding a high risk of resternotomy increases patient comfort and safety.

\section{INTRODUCTION}

The rate of patients being referred for mitral valve surgery after previous cardiac surgery is expected to increase [Casselman 2007]. Although median sternotomy continues to be a common surgical approach, it is technically challenging and associated with complications, including injury to the right ventricle, injury to patent coronary artery bypass grafts, and bleeding [Park 2010]. Additionally, when performing redo mitral valve surgery, exposure can be limited in patients with prior aortic valve replacement or patent right internal mammary artery grafts [Roselli 2008]. Therefore, redo sternotomy may increase operative morbidity and mortality in certain patients.

Received February 4, 2020; received in revised form February 20, 2020; accepted February 20, 2020.

Correspondence: Prof. Dongjin Wang, Department of Thoracic and Cardiovascular Surgery, the Affiliated Drum Tower Hospital of Nanjing University Medical School, 321 Zhongshan Rd, Nanjing 210008, China (e-mail: gldjw@163.com or shuqu_1982@sina.com).
A right infra-axillary incision has been widely used in our center for cosmetic purposes in young people as a good alternative to standard median sternotomy [Wang 2018]. It was first used to repair atrial septal defect, partial atrioventricular septal defect, and ventricular septal defect. With accumulated experience, application of the incision has been consciously extended to mitral valve repair [Wu 2012] and replacement [Wang 2009], and also double valve replacement [Xu 2015]. It can offer excellent exposure of the mitral valve.

Therefore, the authors used a right infra-axillary incision for high-risk patients undergoing mitral valve redo procedures. This article describes our experience using this alternative method.

\section{METHODS}

\section{Patient Enrollment}

From March 2014 to May 2019, 189 patients underwent redo mitral valve surgery at the Department of Thoracic and Cardiovascular Surgery in the Affiliated Drum
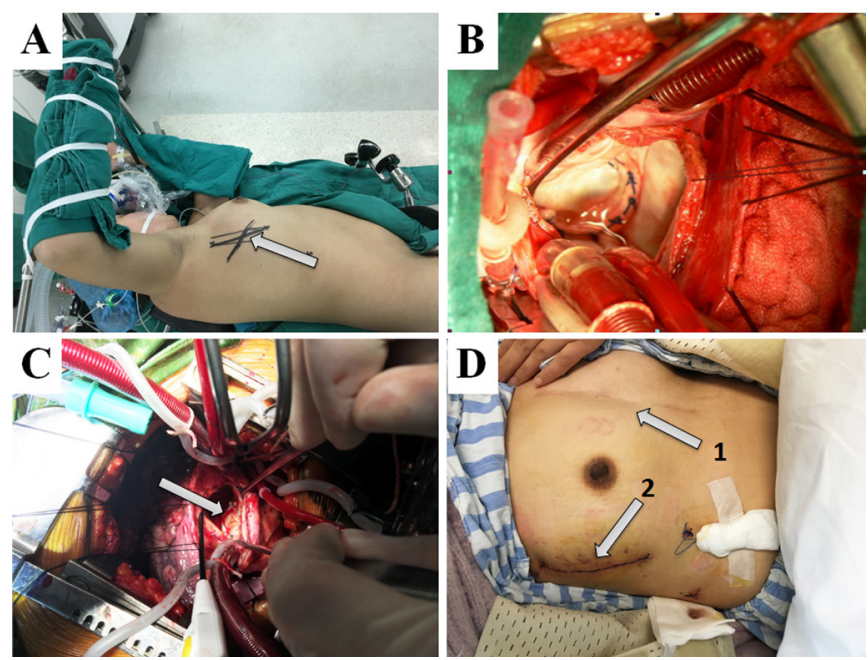

A, Position of patients with the chest in an $80^{\circ} \sim 90^{\circ}$ left lateral position. The bold black line (arrow) between midaxillary and preaxillary line shows where the incision will be made. $B$, Reoperative mitral valve repair can be performed with good exposure. $C$, The exposure of a degenerated bioprosthetic valve (arrow). D, The primary incision (arrow 1 ) and the redo incision (arrow 2). 
Table 1. Patient Demographic and Preoperative Clinical Data*

\begin{tabular}{|c|c|}
\hline Variable & Value \\
\hline Age $(y)$ & $44.4 \pm 6.8$ \\
\hline \multicolumn{2}{|l|}{ Sex } \\
\hline Male & $19(59.4)$ \\
\hline Female & $13(40.6)$ \\
\hline \multicolumn{2}{|l|}{ Previous operation time (mo) } \\
\hline 1 & $29(90.6)$ \\
\hline 2 & $3(9.4)$ \\
\hline \multicolumn{2}{|l|}{ Primary operation } \\
\hline$A V R+M V P$ & 7 \\
\hline MVP & 7 \\
\hline AVR & 6 \\
\hline$M V P+T V P+C A B G$ & 4 \\
\hline$M V R+T V P$ & 3 \\
\hline$A V R+M V R$ & 2 \\
\hline$M V R+C A B G$ & 2 \\
\hline$A V R+C A B G$ & 1 \\
\hline \multicolumn{2}{|l|}{ New York Heart Association class } \\
\hline Class & $8(25.0)$ \\
\hline Class & $19(59.4)$ \\
\hline Class & $5(15.6)$ \\
\hline \multicolumn{2}{|l|}{ Indications } \\
\hline Previous aortic valve replacement & 16 \\
\hline Multiple previous sternotomies & 3 \\
\hline Previous mediastinitis & 4 \\
\hline Recent sternotomy & 1 \\
\hline Previously placed coronary grafts & 7 \\
\hline Severe peripheral vascular disease & 1 \\
\hline Atrial fibrillation & 23 \\
\hline Left ventricular ejection fraction (\%) & $47.9 \pm 13.3$ \\
\hline Left atrial diameter (mm) & $69 \pm 13$ \\
\hline Right atrial diameter (mm) & $52 \pm 14$ \\
\hline EuroSCORE II-predicted mortality (\%) & $12.4 \pm 4.6$ \\
\hline
\end{tabular}

*Data are presented as mean \pm SD or $\mathrm{n}(\%)$. AVR indicates aortic valve replacement; CABG, coronary artery bypass grafting; MVP, mitral valve plasty; MVR, mitral valve replacement; TVP, tricuspid valvuloplasty.

Tower Hospital of Nanjing University Medical School. All the patients had $\geq 1$ prior operation that had been performed via median sternotomy. All gave written informed consent for their detailed clinical data. Computed tomography scanning and angiography were performed for all patients. Right infra-axillary thoracotomy was used in 32 patients, and the others underwent primary median sternotomy. The study
Table 2. Summary of Operative Variables*

\begin{tabular}{lc}
\hline Variable & Value \\
\hline Category of operation & \\
$\quad$ Mitral valve repair & 10 \\
$\quad$ Mitral valve replacement & 22 \\
$\quad$ Left atrial folding & 6 \\
Total surgery time (min) & $206 \pm 73$ \\
Cardiopulmonary bypass time (min) & $86 \pm 18$ \\
Cross-clamp time (min) & $53 \pm 15$ \\
Blood loss during operation (mL) & $226 \pm 128$ \\
Blood transfusion during operation (mL) & $235 \pm 125$ \\
Incision length (cm) & $12.1 \pm 1.7$ \\
\hline
\end{tabular}

*Data are presented as mean \pm SD or $n$.

was conducted according to the Helsinki Declaration and was approved by the ethics committee of Nanjing University.

\section{Indications}

Only 72 patients who had undergone isolated mitral valve surgery and left atrial folding were considered for right infraaxillary thoracotomy. The indications for approaching the mitral valve through the right infra-axillary thoracotomy are (1) previous aortic valve replacement, (2) multiple previous sternotomies, (3) previous mediastinitis, (4) recent sternotomy in which adhesions are likely dense, (5) previously placed bypass graft crossing the midline with a distance $<1 \mathrm{~cm}$ from the posterior surface of the sternum or fixed to the sternum (lack of movement on angiography), and (6) severe peripheral vascular disease causing femoral cannulation to be difficult. Thus 40 low-risk patients received conventional resternotomy.

\section{Operative Technique}

Before draping, an external defibrillator is placed on the patient in case subsequent defibrillation is needed. A double lumen endotracheal tube is used to decompress the right lung. The patient is positioned with the chest in an $80^{\circ} \sim 90^{\circ}$ left lateral position and the pelvis in a corresponding $90^{\circ}$ position. The right arm is over the head, with the shoulder joint abducted approximately $120^{\circ}$ and the elbow joint in a right angle. The skin incision begins at the second intercostal space along the right midaxillary line, extending to the fifth intercostal space along the preaxillary line, which forms a right infra-axillary incision (Figure A). The length of the incision is approximately 9 to $14 \mathrm{~cm}$.

The thoracic cavity is entered through the fourth intercostal space. Two retractors are used to expose the thoracic cavity. The lung is retracted posteriorly using wet sponges to expose the pericardium (Figures B and C). In the case of pleural adhesions resulting from prior surgery, the right lung is dissected from the thoracic wall. The pericardium is opened and dissected $2 \mathrm{~cm}$ anterior to the phrenic nerve, to provide 
Table 3. Postoperative Data of All Patients*

\begin{tabular}{lc}
\hline Variable & Value \\
\hline Drainage the first day $(\mathrm{mL})$ & $233 \pm 63$ \\
Ventilator $>24 \mathrm{~h}$ & $7(21.8)$ \\
Intensive care unit stay $>3 \mathrm{~d}$ & $6(18.8)$ \\
Continuous renal replacement therapy & $1(3.1)$ \\
Extracorporeal membrane oxygenation & $1(3.1)$ \\
Postoperative hospital stay $(\mathrm{d})$ & $11.3 \pm 3.8$ \\
\hline
\end{tabular}

*Data are presented as mean \pm SD or $\mathrm{n}(\%)$.

enough exposure of the ascending aorta and the inferior vena cava together. If the adhesion is too dense, it is not suitable for aortic and vena cava cannulations. In this case, femoral artery and vein cannulation is selected.

To support the removal of air during reperfusion, the surgical field is flushed constantly with CO2 $(5 \mathrm{~L} / \mathrm{min})$. After beginning cardiopulmonary bypass, dissection of the ascending aorta for conventional aortic cross-clamping is initially attempted in all patients. Twenty-six patients underwent cardiopulmonary bypass with mild hypothermia $\left(32^{\circ} \mathrm{C}\right.$ to $\left.34^{\circ} \mathrm{C}\right)$, antegrade cold blood high-potassium cardioplegic arrest. Six patients were cooled to $24^{\circ} \mathrm{C}$, and ventricular fibrillation was induced to perform the surgery under continued retrograde perfusion via coronary sinus after failure to dissect the ascending aorta.

\section{Statistical Analysis}

Statistical analysis was performed using SPSS, version 15.0 (SPSS, Chicago, IL). Continuous variables are expressed as mean \pm standard deviation (SD). Categoric variables are presented as numbers and proportions.

\section{RESULTS}

\section{Patient Characteristics}

The demographic data of the patients are shown in Table 1. The authors studied 32 patients from this series (19 males and 13 females, mean age $44.4 \pm 6.8$ years) who underwent redo mitral valve surgery via right infra-axillary thoracotomy. Three patients had already undergone previous cardiac operations. Table 1 lists all the primary operations. All patients had mitral valve insufficiency; 23 patients had atrial fibrillation; and 8, 19, and 5 patients were New York Heart Association Class II, III, and IV, respectively. The mean left ventricular ejection fraction was $47.9 \%$, and the left and the right atrial diameters were $69 \pm 13$ and $52 \pm 14 \mathrm{~mm}$, respectively. EuroSCORE II-predicted mortality was $12.4 \% \pm 4.6 \%$.

\section{Operative Characteristics}

Table 2 lists the categories of operations. Mitral valve repair and replacement were performed in 10 and 22 patients, respectively, and 6 patients received left atrial folding. The patients were in the operating room for a mean time of 206 minutes, and they had average durations of cardiopulmonary bypass and cross-clamp of 86 and 53 minutes, respectively. Mean blood loss during the operations was $226 \pm 128 \mathrm{~mL}$, and mean blood transfusion volume was $235 \pm 125 \mathrm{~mL}$. Mean incision length was $12.1 \pm 1.7 \mathrm{~cm}$ (Figure D). The intraoperative course was uneventful, and no patient was converted to a full sternotomy.

\section{Outcomes and Follow-Up}

Postoperative data are shown in Table 3. Mean chest drainage volume of the first 24 hours was $233 \pm 63 \mathrm{~mL}$, and there was no postoperative blood transfusion in 25 patients. In 7 patients, duration of mechanical ventilation exceeded 24 hours, and in 6 patients, intensive care unit stay exceeded 3 days. One patient received continuous renal replacement therapy for oliguresis. Extracorporeal membrane oxygenation was performed in 1 patient for low output syndrome. No patient died in the hospital; mean postoperative stay in the hospital was $11.3 \pm 3.8$ days.

Postdischarge information was obtained by clinical followup and telephone interview. The follow-up duration ranged between 6 and 71 months, and the follow-up rate was $100 \%$. All patients were surviving at the time of follow-up, and they were willing to personally provide information regarding their functional status. There were no late deaths or cardiovascular accidents during follow-up.

\section{DISCUSSION}

Redo procedures are associated with increased perioperative risk. In the past, redo mitral valve operations were usually performed through median sternotomy, with high risk and mortality. With further development of surgical techniques, minimally invasive redo mitral valve surgery has become reliable, with results comparable to those of conventional techniques [Casselman 2007, Bolotin 2004, Patel 2017]. Therefore, there is great interest in less invasive and safer approaches to heart surgery, especially when these alternative access routes decrease surgical risk and do not compromise quality. Because of these findings and their experience with the right infra-axillary incision, the authors have performed redo mitral valve surgery via right infraaxillary incision in high-risk patients. This series documents 32 patients who underwent the less-invasive technique for redo mitral valve procedures.

The principal advantage of the right infra-axillary incision is, first, injury reduction. It obviates the need to divide or incise muscles other than the intercostal muscles, preserves the long thoracic nerve, retains a normal space between the ribs, and does not hinder the symmetrical development of the breasts or nipple sensitivity. Our current data have not reported any major hemorrhage or mortality associated with dissection of the adhesions via the right infra-axillary incision. The incision is in the chest wall, which has low muscle mass and is far from the costochondral junction; it does not 
interfere with the development of the chest wall, particularly in young patients. In addition, femoral cannulation is not necessary [Casselman 2007, Wang 2018], thus it also avoids femoral artery injury.

Second, this incision offers excellent visualization of mitral valve structures in a direct-line view. Moreover, it is easy to learn and practice compared with other minimally invasive approaches, thoracoscopy, or robotic technology [Bolotin 2004, Patel 2017, Cao 2017, Holzhey 2013]. It is mostly similar to the median sternotomy. Its access can provide enough exposure of the ascending aorta and the venae cavae, as well as improve visualization of the atria and the mitral valve, which can be examined under direct vision. The use of a straight arterial cannula, the procedure, and cannulation are similar to those of median sternotomy.

Third, the incision does not require special surgical instruments, and it does not increase costs. The entire procedure is performed under direct vision without additional devices, such as video-assisted thoracoscopic equipment. The incision may be one of the most economical and practical procedures compared with other minimally invasive approaches, the thoracoscopy [Bolotin 2004], and robotic technology [Patel 2017]. Onnasch [2002] described redo mitral valve surgery by right thoracotomy, which is a good alternative, but it requires special instrumentation such as the Chitwood/Cosgrove clamp, a knotter/pusher, and occasionally thoracoscopic assistance. The requirements of special instrumentation and the use of femoral artery or vein cannulation slightly increase the cost, and the incision may injure the pectoral muscles and mammary tissue. Also, it has an effect on the frontal view and shape of the breast.

Finally, the authors have found that obesity, increased age, or reduced heart function are not contraindications for this procedure. This incision is associated with significantly fewer blood transfusions and less chest drainage, most likely from avoiding more extensive and time-consuming dissection of adhesions, which also might contribute to the quick recovery. As shown in our follow-up data, the use of the right infraaxillary incision did not increase the incidence of the early complications, and the visual analogue scale score used to measure postoperative pain did not indicate that the spreading of the ribs was more painful than in a sternotomy [Yaliniz 2015, Luo 2014]. Furthermore, this incision did not affect the results of the coughing exercise after surgery, which is beneficial for early rehabilitation.

The incidence of intraoperative injury to cardiac structures has been reported to occur in $5 \%$ to $10 \%$ of resternotomies [Park 2010, Roselli 2008]. The most common injured structures were patent coronary graft $(46 \%)$ and right ventricle (21\%). Although operative mortality might not be considered with intraoperative injury in some studies [Ellman 2008, Ghoreishi 2013], most studies showed that overall mortality was significantly higher if reentry injury occurred [Park 2010, Roselli 2008]. Therefore, several strategies have been described as safe alternatives to the conventional redo sternotomy, including the right anterolateral thoracotomy approach [Onnasch 2002], thoracoscopy [Bolotin 2004], the roboticassisted approach [Patel 2017], and transcatheter mitral valve implantation [Keenan 2019]. Durations of the operation and hospitalization can be used as a benchmark. These alternative strategies would entail a substantially longer operative time, including a longer time on bypass and cross-clamp. Moreover, they offer little benefit over the open technique in terms of length of hospitalization or intensive care unit stay. Although transcatheter mitral valve implantation is feasible, safe, and efficacious, it has poor durability data and a high cost limit for its clinical application, and thus is for wealthy, high-risk older patients [Keenan 2019].

The sternotomy can be used to perform various cardiovascular surgeries. It conforms to the habits of the cardiac surgeon. It can be handled more safely in the event of accidents, and myocardial protection can be better using sternotomy. Therefore, repeating the sternotomy is the first-choice operation for most patients with a previous sternotomy. In the present study, 117 patients were receiving more than mitral valve surgery and left atrial folding; thus resternotomy was chosen. The safety of repeating the sternotomy mitral valve operation in low-risk patients with low perioperative mortality and morbidity and low rates of intraoperative injury has been proven [Ghoreishi 2013, Patel 2019]. The authors recommended that the 32 high-risk patients receive the mitral valve surgery via the right infra-axillary thoracotomy. As this incision requires surgeons with significant experience performing this incision, and it causes a new scar, the other 40 low-risk patients were operated via resternotomy by a young surgeon for training.

\section{Limitations}

First, our patient population is small because of the rarity of patients who require redo mitral valve surgery with a high-risk resternotomy. Second, this study was based on retrospective information from our center database, and some variables, such as the length of intensive care unit stay and hospital stay, were determined largely by the speed of the patient's recovery from surgery, which can be influenced by subjective factors. In addition, a postoperative assessment of quality of life was not performed, and a comparison between the right infra-axillary incision and the median thoracotomy or other incisions was not undertaken.

\section{Conclusion}

With rich experience performing the right infra-axillary incision in our center, it has become an effective approach for high-risk redo mitral valve procedures. It can be performed safely and can reduce the possibility of injury to cardiac structures. Avoiding a resternotomy may decrease the perioperative risk for patients, and it could increase comfort for them after redo mitral valve surgery. This procedure is familiar to cardiac surgeons, and it is easy to grasp after practicing on corrected, relatively simple cardiac defects.

\section{ACKNOWLEDGMENTS}

We thank Ye Li for collecting clinical data for this study. This work was supported in part by Jiangsu Provincial Medical 
Youth Talent (QNRC2016034), Jiangsu Province Health Department Program Grant (Z201411), and Key Project supported by Medical Science and Technology Development Foundation, Nanjing Department of Health (YKK17066).

\section{REFERENCES}

Bolotin G, Kypson AP, Reade CC, et al. 2004. Should a video-assisted mini-thoracotomy be the approach of choice for reoperative mitral valvesurgery? J Heart Valve Dis 13:155-158.

Cao H, Zhou Q, Fan F, Xue Y, Pan J, Wang D. 2017. Right anterolateral thoracotomy: An attractive alternative to repeat sternotomy for high-risk patients undergoing reoperative mitral and tricuspid valve surgery. J Cardiothorac Surg 12:85.

Casselman FPCasselman FP, La Meir MLa Meir M, Jeanmart HJeanmart H, et al. 2007. Endoscopic mitral and tricuspid valve surgery after previous cardiac surgery. Circulation 116:I270-I275.

Ellman PI, Smith RL, Girotti ME, et al. 2008. Cardiac injury during resternotomy does not affect perioperative mortality. J Am Coll Surg 206:993-997.

Ghoreishi M, Dawood M, Hobbs G, et al. 2013. Repeat sternotomy: No longer a risk factor in mitral valve surgical procedures. Ann Thorac Surg 96:1358-1365.

Holzhey DM, Seeburger J, Misfeld M, Borger MA, Mohr FW. 2013. Learning minimally invasive mitral valve surgery: A cumulative sum sequential probability analysis of 3895 operations from a single highvolume center. Circulation 128:483-491.

Keenan NM, Bennetts JS, McGavigan AD, et al. 2019. Transcatheter transseptal mitral valve-in-valve replacement: An early Australian case series and literature review. Heart Lung Circ S1443-9506:31373-31373.

Luo H, Wang J, Qiao C, Zhang X, Zhang W, Song L. 2014. Evaluation of different minimally invasive techniques in the surgical treatment of atrial septal defect. J Thorac Cardiovasc Surg 148:188-193.

Onnasch JF, Schneider F, Falk V, Mierzwa M, Bucerius J, Mohr FW. 2002. Five years of less invasive mitral valve surgery: From experimental to routine approach. Heart Surg Forum 5:132-135.

Park CB, Suri RM, Burkhart HM, et al. 2010. Identifying patients at particular risk of injury during repeat sternotomy: Analysis of 2555 cardiac reoperations. J Thorac Cardiovasc Surg 140:1028-1035.

Patel H, Lewis CTP, Stephens RL, Angelillo M, Sibley DH. 2017. Minimally invasive redo mitral valve replacement using a robotic-assisted approach. Innovations (Phila) 12:375-377.

Patel NC, Hemli JM, Seetharam K, et al. 2019. Reoperative mitral valve surgery via sternotomy or right thoracotomy: A propensity-matched analysis. J Card Surg 34:976-982.

Roselli EE, Pettersson GB, Blackstone EH, et al. 2008. Adverse events during reoperative cardiac surgery: Frequency, characterization, and rescue. J Thorac Cardiovasc Surg 135:316-323.

Wang D, Wang Q, Yang X, Wu Q, Li Q. 2009. Mitral valve replacement through a minimal right vertical infra-axillary thoracotomy versus standard median sternotomy. Ann Thorac Surg 87:704-708.

Wang Q, Ye JX, Ge M, Wang DJ. 2018. Early- and long-term outcomes of cardiovascular surgery via minimal right vertical infra-axillary thoracotomy: A 15-year study of 1,126 patients. Sci Rep 28:4376.

Wu Z, Pan J, Wang Q, Zhou Q, Wang D. 2012. Surgical repair of mitral valve prolapse through a minimal right vertical infraaxillary thoracotomy. J Card Surg 27:533-537.

Xu C, Pan J, Zhou Q, et al. 2015. Right vertical infra-axillary thoracotomy for double valve replacement. J Card Surg 30:427-430.

Yaliniz H, Topcuoglu MS, Gocen U, et al. 2015. Comparison between minimal right vertical infra-axillary thoracotomy and standard median sternotomy for repair of atrial septal defects. Asian J Surg 38:199-204. 